DOI: https://doi.org/10.30749/2594-8261.v5n3p77-93

\title{
RELATO SOBRE PRÁTICAS PEDAGÓGICAS: PROFESSORES COMPARTILHANDO SUAS EXPERIÊNCIAS NO PERÍODO DA PANDEMIA COVID-19
}

\section{REPORT ON PEDAGOGICAL PRACTICES: TEACHERS SHARING THEIR EXPERIENCES IN THE COVID-19 PANDEMIC PERIOD}

\author{
André Machado Barbosa ${ }^{1}$ \\ Marco Antonio Serra Viegas ${ }^{2}$ \\ Marco Aurelio Carvalho da Costa ${ }^{3}$ \\ Regina Lucia Napolitano F. F. Batista ${ }^{4}$
}

\begin{abstract}
Resumo: O trabalho é resultante de uma pesquisa norteada pelo método de observação, a qual tem como abordagem o relato das experiências pedagógicas, nesse período da pandemia da COVID-19, de quatro profissionais de educação, que atuam em segmentos diferenciados. Tem como objetivo compartilhar as experiências didáticas dos quatro autores nos respectivos segmentos para sociedade em geral, principalmente a acadêmica. Os relatos compreendem práticas pedagógicas do ensino básico, inclusive, na modalidade de Educação Especial, Educação de Jovens e Adultos (EJA) e Instituições de Ensino Superior (IES). Em suas considerações finais, após suas narrações de observação, confluem, como resultado, sobre a necessidade de estarem atentos ao habitual e às atualizações pelas quais as instituições de ensino e a sociedade estão passando. Esses profissionais têm como princípio norteador desenvolverem-se ainda mais, pois uma vez professor, eternamente estudante; e assim, podem oferecer práticas pedagógicas e transmissão do conhecimento de forma eficaz, eficiente e efetiva. É fato que sempre haverá necessidade, no exercício da profissão, de aprimorarem as competências, a fim de desenvolverem habilidades e atitudes nos estudantes, o que demonstrará a eficiência de suas capacidades profissionais.
\end{abstract}

Palavras-chave: Conteúdos Educacionais. Prática Pedagógica. Tecnologia. Metodologias ativas.

\footnotetext{
${ }^{1}$ Pesquisador e Professor, Mestre em Desenvolvimento Local (DL), Administração, UFRJ/LAMAE, UNESA/GEPA, Faculdade Signorelli, e-mail: andre.mb.adm@gmail.com

2 Pesquisador e Professor, Mestrando em Educação, UFRRJ/LEPEDI, e-mail: srmarcoviegas@gmail.com

${ }^{3}$ Pesquisador e Professor, Letras, UERJ, e-mail: marcoaureliocosta69@gmail.com

${ }^{4}$ Professora e Pesquisadora, Mestre em Ciências Pedagógicas e em Desenvolvimento Local (DL), Tec. da Informação, Pedagogia, UFRJ/LAMAE, CEDERJ, IFRJ, UNESA/GEPA, e-mail: reginafelicio01@gmail.com
} 
RELATO SOBRE PRÁTICAS PEDAGÓGICAS: PROFESSORES COMPARTILHANDO SUAS EXPERIÊNCIAS NO PERÍODO DA PANDEMIA COVID-19
André Machado Barbosa Marco Antonio Serra Viegas Marco Aurelio Carvalho da Costa Regina Lucia Napolitano F. F. Batista

Abstract: The work is the result of a research guided by the observation method, which has as an approach the report of pedagogical experiences, in this period of the COVID-19 Pandemic, of four education professionals who work in different segments. Its objective is to share the didactic experiences of the four authors in their respective segments for society in general, mainly academic. The reports comprise pedagogical practices of basic education, including in the form of Special Education, Youth and Adult Education (EJA) and Higher Education Institutions (IES). These professionals have as their guiding principle to develop even more, because once a teacher, they are eternally a student; and thus, they can offer pedagogical practices and knowledge transmission in an effective, efficient and effective way. It is a fact that there will always be a need, in the exercise of the profession, to improve skills to develop skills and attitudes in students, which will demonstrate the efficiency of their professional skills.

Keyword: Educational Content. Pedagogical Practice. Technology. Active methodologies.

Recebido em: 07/01/2022 Aceito para publicação em: 16/01/2022 
RELATO SOBRE PRÁTICAS PEDAGÓGICAS: PROFESSORES COMPARTILHANDO SUAS EXPERIENNCIAS NO PERÍODO DA PANDEMIA COVID-19
André Machado Barbosa Marco Antonio Serra Viegas Marco Aurelio Carvalho da Costa Regina Lucia Napolitano F. F. Batista

\section{INTRODUÇÃO}

No momento atual em que o mundo passa pela situação pandêmica com o coronavírus, todos foram obrigados a mudar sua rotina de trabalho, estudos, lazer e outras. E claro, o espaço escolar foi profundamente impactado. As instituições de ensino tiveram suas estruturas físicas fechadas por mais de um ano, o que provocou a suspensão das aulas no modelo tradicional. Com isso, logo no primeiro momento, surge um novo modelo de ensino denominado híbrido, que, por meio remoto, a metodologia tornou possível aos estudantes o acesso a conteúdos com a mediação do educador em tempo real, ou seja, online.

Assim sendo, com todos os envolvidos no processo de aprendizado dos estudantes, houve necessidade de conhecer e aprimorar-se em tecnologias que propiciassem a apresentação dos conteúdos escolares. Envolvidos na missão, as organizações adaptaram o trabalho pedagógico com os estudantes, assim como a avaliação dos tópicos expostos nos ambientes virtuais de aprendizagem.

Dado aos fatos, buscamos, através do referencial teórico, demonstrar o processo de ensino-aprendizagem aplicado e relatado nas experiências dos educadores envolvidos na pesquisa, utilizando o método de observação. Constantes reflexões permeiam o universo estudantil no que se refere aos resultados obtidos na aplicabilidade das aulas de forma síncrona no ambiente tecnológico, aos conteúdos educacionais sobrepostos nessa nova modalidade de ensino e ao treinamento para o aperfeiçoamento profissional dos educadores, sempre protagonistas, e agora com as novas metodologias e métodos, precisam, cada vez mais, utilizarem metodologias ativas para dinamizar o processo a fim de atrair a atenção dos estudantes.

Em razão dessas considerações, a finalidade deste artigo está em compartilhar conhecimento, por meio das experiências didáticas dos quatro autores nos respectivos segmentos: Educação Básica, Educação Especial, Educação de Jovens e Adultos e Educação no Nível Superior. Em suas considerações finais, os autores confluem sobre a necessidade de estarem atentos às práticas pedagógicas, 
RELATO SOBRE PRÁTICAS PEDAGÓGICAS: PROFESSORES COMPARTILHANDO SUAS EXPERIENNCIAS NO PERÍODO DA PANDEMIA COVID-19
André Machado Barbosa Marco Antonio Serra Viegas Marco Aurelio Carvalho da Costa Regina Lucia Napolitano F. F. Batista

ao habitual e às atualizações pelas quais instituições de ensino e sociedade estão passando.

\section{METODOLOGIA}

A metodologia usada neste trabalho é uma pesquisa descritiva, em que procuramos relatar nossas experiências como professores de diferentes níveis e modalidades de educação, de forma longitudinal, utilizando o método de observação. O campo explorado, portanto, foi os espaços onde os educadores trabalham.

Martino (2018, p. 135), sobre essa prática de pesquisa de campo, descreve como definição de observação "A alegria de olhar" e inclusive faz referência ao primeiro parágrafo de Metafísica, um conjunto de tratados aristotélicos, onde o grande filosofo grego escrevia que "todos os seres humanos desejam, por natureza, do saber". E continua: "Prova disso é a alegria que temos de olhar para as coisas".

$\mathrm{Na}$ narrativa da pesquisa, pelo viés metodológico qualitativo, buscou-se um referencial teórico dada as vivências relatadas para uma coesão textual. Logo, buscou-se retratar suas vivências em caráter exploratório de campo, com o objetivo de contribuírem para a sociedade acadêmica com esse relato de experiências.

\section{REFERENCIAL TEÓRICO}

\subsection{Conteúdos Educacionais}

É possível definir conteúdos educacionais como materiais que aspiram a levar conhecimento e aprendizado a um determinado público, assim como valores e juízos. Assim, Filatro (2018, p. 21) nos faz entender que, para elaborar a apresentação de conteúdo ou a proposição de atividades para uma unidade de estudo, é imprescindível determinar previamente o que se pretende realizar na promoção de um aprendizado específico. 
RELATO SOBRE PRÁTICAS PEDAGÓGICAS: PROFESSORES COMPARTILHANDO SUAS EXPERIENNCIAS NO PERÍODO DA PANDEMIA COVID-19
André Machado Barbosa Marco Antonio Serra Viegas Marco Aurelio Carvalho da Costa Regina Lucia Napolitano F. F. Batista

Para nossa discussão, especialmente, os conteúdos educacionais são direcionados a docentes e discentes e sua produção em modo eletrônico, postado nas plataformas digitais de conhecimento. Fato este que, neste período de crise global, com a pandemia do coronavírus, fortaleceu a relação tecnológica entre educadores e estudantes, bem como ensino e aprendizagem, para garantir a construção do conhecimento, com intuito de desenvolver habilidades.

A produção de conteúdos educacionais significa, na prática, o ato de ofertar instrumentos de qualidade em diversos modelos impressos ou digitais que atendam ao público-alvo com relevância. Talbert (2019, p. 91) nos coloca a importância da preparação dos conteúdos, ao afirmar que, somente depois de feito o design completo de uma disciplina, estamos prontos para redigir um plano de ensino e preparar as aulas individualmente.

Logo, a apresentação, produção e administração de conteúdos educacionais perpassam pela pesquisa sobre os temas a fim de servirem como guia de um plano de aula para que os docentes, com suas habilidades, apliquem um bom e adequado método de transmissão. Autores como Darling-Hammond e Bransford (2019 p. 77) afirmam que "ensinar não é só falar, e aprender não é apenas ouvir." Destacam a importância de os educadores não apenas transmitirem conteúdo, mas também fazê-lo de modo que os estudantes possam entender e utilizar esses conhecimentos, transformando-os em habilidades.

\subsection{Prática Pedagógica}

A prática pedagógica dá-se muito além da prática didática, apesar de serem muito confundidas. A prática pedagógica compreende o contexto da formação, os espaços-tempo escolares, as possibilidades de organização do trabalho docente, as parcerias e expectativas.

Sobre a prática pedagógica, cabe a compreensão que se referem a práticas sociais que são exercidas com a finalidade de concretizar processos pedagógicos. Posto isto, Darling-Hammond e Bransford (2019, p. 79) nos fazem refletir ao afirmar 
RELATO SOBRE PRÁTICAS PEDAGÓGICAS: PROFESSORES COMPARTILHANDO SUAS EXPERIENNCIAS NO PERÍODO DA PANDEMIA COVID-19
André Machado Barbosa Marco Antonio Serra Viegas Marco Aurelio Carvalho da Costa Regina Lucia Napolitano F. F. Batista

que, quando o desenvolvimento do estudante é o foco das decisões da prática docente, os professores planejam de acordo com as necessidades de seus alunos e dão suporte a sua progressão ao longo de várias rotas desenvolvimentais - físicas, sociais, emocionais, cognitivas, linguísticas e psicológicas.

Portanto, as práticas pedagógicas desempenham $\circ$ papel de organizar/potencializar/interpretar os propósitos de um projeto educativo. Essas práticas, em todos os níveis e modalidades da Educação, desde a educação básica ao ensino superior, é de grande relevância na constituição dos sujeitos envolvidos em todo o processo de escolarização.

\subsection{Tecnologia}

A tecnologia é uma grande parceira da Educação, que possibilita a todos, com destaque para professores e alunos, estar em contato com novas ferramentas de aprendizagem, seja nos espaços profissionais ou nos ambientes educacionais, oferecendo recursos para um processo de aprendizado flexível e dinâmico. Cortelazzo (2018, p. 171) nos traz a reflexão de que, assim, podemos ver claramente uma relação entre escolas, universidades e ambientes de trabalho inovadores e aprender muito com eles, o que nos faz pensar a relação próxima que a tecnologia traz aos diversos mundos.

Há compreensão sobre o papel da tecnologia na Educação ir além dos limites das salas de aula virtuais ou plataformas de ensino. Nesse processo de transformação de realidades dos docentes e discentes, novas experiências e uma nova forma de ensinar e se relacionar com o conhecimento surgem e podem demonstrar ser objeto de transformação social. Carvalho, Borges e Ameno (2018, p. 97) contribuem neste aspecto, pois falam do papel da escola em criar mecanismos para os estudantes tornarem-se criativos e inovadores, levando-os a desenvolver habilidades cognitivas de inovar por meio do senso crítico.

O uso da tecnologia foi fundamental para continuidade desse processo de ensino-aprendizagem e denota como as instituições de ensino, principalmente as 
RELATO SOBRE PRÁTICAS PEDAGÓGICAS: PROFESSORES COMPARTILHANDO SUAS EXPERIENNCIAS NO PERÍODO DA PANDEMIA COVID-19
André Machado Barbosa Marco Antonio Serra Viegas Marco Aurelio Carvalho da Costa Regina Lucia Napolitano F. F. Batista

pioneiras, foram eficazes nas tomadas de decisão. Ao adotarem o modelo híbrido, essas instituições contribuíram para a transformação da realidade educacional e viabilizaram a continuidade das aulas.

Deste modo, podemos refletir nas inúmeras possibilidades que a tecnologia nos abraça com seu advento e sua utilização nos diversos espaços e eventos do nosso cotidiano. Na viabilidade de tornar nossas realidades imaginárias possíveis, podemos refletir sobre a contribuição dos recursos tecnológicos, a eficácia e eficiência alcançada, na prática do ensinar e aprender com troca de vivências entre educadores e estudantes.

\subsection{Metodologias Ativas}

Educadores a todo momento foram desafiados a constante busca por estratégias didáticas inovadoras, a fim de tornarem suas aulas mais criativas e dinâmicas. Mas, é claro, devido ao cenário pandêmico, isso foi acirrado, o que provocou certo desconforto para alguns, assim como oportunidades para vários.

No ambiente de sala de aula presencial, com o objetivo de melhorar a compreensão dos estudantes, as metodologias ativas vão além dessas expectativas para os educadores, porque, além de facilitar o processo de ensino-aprendizagem, torna-os sujeitos autônomos. Cabe ressaltar que, no caso das aulas remotas, ou seja, no ambiente síncrono, a finalidade amplia, porque, além da necessidade de entendimento por parte dos estudantes, seu uso faz-se necessário para dinamizar e atrair a atenção, como também realizar a interação entre eles.

Segundo Moran (2018, p. 4), as metodologias ativas contribuem no processo ensino-aprendizagem, enfatizam o papel protagonista dos estudantes, permitindo seu envolvimento direto, participativo e reflexivo em todo esse processo, com a orientação dos professores. Tais práticas tornam o educador criativo, um professor com potencial inovador e não apenas um mero transmissor de conteúdo, pois aguçam a curiosidade dos estudantes e os levam a aprender com intensidade. 
RELATO SOBRE PRÁTICAS PEDAGÓGICAS: PROFESSORES COMPARTILHANDO SUAS EXPERIENNCIAS NO PERÍODO DA PANDEMIA COVID-19
André Machado Barbosa Marco Antonio Serra Viegas Marco Aurelio Carvalho da Costa Regina Lucia Napolitano F. F. Batista

O ambiente educacional, neste período, com auxílio das ferramentas tecnológicas, possibilitou práticas inovadoras de ensino, ampliando o modelo síncrono nas universidades do nosso país. Sendo assim, revolucionou a sala de aula de certa forma, dado o desafio de acolher e potencializar os estudantes aplicando as técnicas de metodologias ativas nesse ambiente virtual.

Leal, Miranda e Nova (2018), cujo livro já em seu título traz essa abordagem da necessidade de revolucionarmos as salas de aulas, foram extremamente precisos em suas colocações, sobretudo em relação a triple helice. Um modelo reconhecido internacionalmente que tem a universidade como ambiente necessário a atender não só as demandas no mundo corporativo e governamental, treinando profissionais preparados para desenvolver a economia, mas também muitos dos 17 Objetivos do Desenvolvimento Sustentável - ODS - (acordo realizado por diversas nações na Conferência das Nações Unidas sobre desenvolvimento sustentável no Rio de Janeiro em 2012, o qual nosso país é signatário).

Sobre a contribuição das metodologias ativas, recorremos a Filatro e Cavalcanti (2018, p. 19). As autoras destacam que podem ser adotadas para desenvolver as competências relevantes do século $\mathrm{XXI}$, sob a perspectiva da pedagogia, da andragogia e da heutagogia. São abordagens muito pertinentes, principalmente a heutagogia, como resposta às demandas da tecnologia digital que chegaram, de forma súbita, diante da pandemia da COVID-19. Em vista dessas, dentre muitas outras implicações, dá-se a seguir os relatos dos professores envolvidos nesta pesquisa.

\section{RESULTADOS E DISCUSSÃO}

\subsection{Professor da Educação Especial}

De acordo com Moran (2018, p. 9), “as pesquisas atuais nas áreas da educação, psicologia e neurociência comprovam que o processo de aprendizagem é único e diferente para cada ser humano". Dessa forma, é fundamental que o 
RELATO SOBRE PRÁTICAS PEDAGÓGICAS: PROFESSORES COMPARTILHANDO SUAS EXPERIENNCIAS NO PERÍODO DA PANDEMIA COVID-19
André Machado Barbosa Marco Antonio Serra Viegas Marco Aurelio Carvalho da Costa Regina Lucia Napolitano F. F. Batista

professor analise as especificidades de cada contexto, sobretudo quando se trata de Educação Especial, para que possibilite a ampliação de conhecimentos e o desenvolvimento de habilidades de seus alunos.

$\mathrm{Na}$ escola em que foi feita a observação, as adequações curriculares das disciplinas ministradas pelos professores da classe regular são confeccionadas pelo professor da sala de recursos multifuncional - AEE (Atendimento Educacional Especializado). Esse profissional trabalha em conjunto com os demais professores, na promoção de tornar os conteúdos possíveis ao aprendizado dos alunos com deficiências.

Neste período de aulas remotas, trazer a tecnologia para a educação especial tem sido um grande empreendimento, que necessita principalmente da colaboração e empenho das famílias, para que ocorra a aprendizagem dos estudantes. Fato que demonstra o quão é relevante a relação família - escola - professor. Desse modo, é importante a relação entre os envolvidos no caminhar do processo pedagógico do sujeito, percebendo a necessidade deles para que o aprendizado aconteça.

Como professor do AEE, na adaptação dos conteúdos escolares, foram utilizados vídeos gravados pelo professor, com materiais reciclados e de fácil compreensão, postados em plataforma de ensino disponível aos alunos, pensando na possibilidade dos responsáveis em realizar as atividades apresentadas com instrumentos acessíveis e comuns a todos. E com essa proposta, no que se refere ao trabalho nas escolas de hoje, os professores devem estar preparados para ensinar na diversidade, pois os alunos são e sempre serão diferentes entre si de várias formas.

O retorno de uma parte dos responsáveis, através de vídeos gravados com os alunos concluindo as atividades, foi um feedback de grande valor, pois confirmou a prática proposta e seu sucesso na execução e inclusão de tecnologias digitais na educação especial, logo, avaliado pela equipe, como ponto positivo. E diante desse fato, faz-se necessário pensar em propostas que possam trazer para a educação uma perspectiva que vise à formação de sujeitos capacitados a agir regularmente de 
RELATO SOBRE PRÁTICAS PEDAGÓGICAS: PROFESSORES COMPARTILHANDO SUAS EXPERIENNCIAS NO PERÍODO DA PANDEMIA COVID-19
André Machado Barbosa Marco Antonio Serra Viegas Marco Aurelio Carvalho da Costa Regina Lucia Napolitano F. F. Batista

maneira digital, caracterizando também como metodologias ativas aplicadas nesse segmento educacional.

\subsection{Professora na Educação de Jovens e Adultos}

Na Educação de Jovens e Adultos, ambiente de cenários muito diferentes, foram analisadas turmas do ensino fundamental segundo segmento, ensino médio técnico e o ensino médio técnico na educação de jovens e adultos. Foram inúmeros os desafios pelo ambiente tecnológico e pelas limitações dos estudantes com a ferramenta.

No ensino fundamental, a realidade é da rede privada e, nos outros, da rede pública. No contexto analisado, tanto os estudantes da rede privada quanto os da rede pública possuem acesso a recursos tecnológicos. Aos estudantes da rede pública foram ofertados incentivos a recursos tecnológicos, como chip, celular e tablet.

A percepção enquanto docente foi de desinteresse e desmotivação de muitos em participar das atividades propostas e utilizar os materiais. Isso ocorreu em ambos os espaços, ou seja, público e privado. Desse modo, buscou-se novas propostas por meio de levantamento de dados, de quais recursos estavam mais próximos à realidade desses estudantes, e uma metodologia que despertasse interesse neles para utilização dos recursos, a fim de realizarem as atividades de acordo com que afirma Moran (2018, p. 4) a respeito das práticas de metodologias ativas.

Foi criado um repositório para postar os conteúdos antes do horário das aulas. Além disso, foi disponibilizado um formulário para registro da frequência, no qual o estudante precisava apresentar um resumo do conteúdo estudado, com suas palavras e realizar as atividades de fixação, em que vai somando os pontos até $50 \%$ para avaliação.

Uma agenda foi montada para os encontros síncronos, cuja duração era de, no mínimo, 40 minutos e, no máximo, 60 minutos. $O$ aluno, neste encontro, tem a 
RELATO SOBRE PRÁTICAS PEDAGÓGICAS: PROFESSORES COMPARTILHANDO SUAS EXPERIENNCIAS NO PERÍODO DA PANDEMIA COVID-19
André Machado Barbosa Marco Antonio Serra Viegas Marco Aurelio Carvalho da Costa Regina Lucia Napolitano F. F. Batista

possibilidade de colocar os pontos que mais the chamaram atenção ou que os deixou em dúvidas sobre o conteúdo.

Em paralelo, a turma possui um grupo de WhatsApp da disciplina, para, a qualquer momento que surgirem dificuldades, ser orientada na resolução de dúvidas ou interações sobre a disciplina. Quanto às avaliações, são ofertados 02 formulários, de 10 questões cada, que contemplam os conteúdos.

Assim, os estudantes têm a oportunidade de acompanhar os conteúdos e serem avaliados, com o propósito de não perderem o período letivo. Entretanto, para essa oportunidade se consumar, o estudante precisa ter acessado o mínimo de 60\% do conteúdo ofertado no repositório. Na avaliação qualitativa, devido ao uso de critérios da gamificação, eles se mais motivados e engajados a participar, uma vez que vão recebendo "itens". Dessa forma, tanto o aprendizado como o desempenho dos alunos nos cenários relatados apresentaram melhor rendimento e maior envolvimento, com a redução em $90 \%$ na insatisfação e na perda do período escolar.

\subsection{Professor do Fundamental II}

Atuando como professor de redação para alunos do fundamental II em uma escola de classe média alta na cidade do Rio de Janeiro, presume-se, nesse cenário, que os efeitos deletérios causados pela pandemia teriam sido menores para essa faixa social, visto que se trata de alunos com maior poder aquisitivo e que teoricamente teriam mais facilidade de acesso aos ambientes virtuais graças a aparelhos mais eficientes e a uma conexão de melhor qualidade. Essa noção é, contudo, superficial e adiantada frente à realidade.

O ensino remoto suscitou novas contingências que, até então, não eram pensadas - uma delas diz respeito ao compartilhamento de conteúdo e à interação entre alunos e professores num ambiente virtual; a outra conta com mudanças nos próprios hábitos, posto que tiveram de inserir o trabalho, antes externo, na rotina 
RELATO SOBRE PRÁTICAS PEDAGÓGICAS: PROFESSORES COMPARTILHANDO SUAS EXPERIENNCIAS NO PERÍODO DA PANDEMIA COVID-19
André Machado Barbosa Marco Antonio Serra Viegas Marco Aurelio Carvalho da Costa Regina Lucia Napolitano F. F. Batista

doméstica. Temos aqui fatos distintos, mas que se entrecruzam enormemente, um influenciando o outro.

Nesse espaço escolar, a plataforma utilizada para o compartilhamento de material e interação foi o Google Classroom. Todo processo foi bastante corrido, e o aprendizado básico dos recursos deu-se concomitante ao uso, dentre o uso de metodologias ativas disponíveis nesse processo, com intuito de dinamizar as aulas.

Durante quase todo o ano de 2020, observei majoritariamente duas posturas das turmas em relação às aulas - uma primeira associada à baixa adesão presencial e uma segunda, de adesão nula. Os horários dos encontros obedeciam à grade normal da escola. Na plataforma, o conteúdo era disposto segundo as escolhas de cada professor - vídeos gravados, Power Point etc. Como método de ensino, optou-se por gerar resumos teóricos em PDFs, para que os alunos pudessem dispor do conteúdo organizado, de forma hierarquizada, ao longo dos meses que se sucederiam.

Para os debates que antecedem as propostas de escrita, o YouTube e links de matérias selecionadas na web foram os recursos mais usados. O principal desafio era conseguir reter online os poucos alunos presentes e fazê-los interagir por chat ou, em raros momentos, por áudio, tendo em vista que sempre se mostraram refratários à interação visual pela câmera, seguindo as tendências do uso da tecnologia conforme Carvalho, Borges e Ameno (2018, p. 97) informa, disposto no referencial, na abordagem sobre tecnologias no presente trabalho.

Quanto ao processo avaliativo, a instituição onde trabalho determinou que as redações deveriam ser manuscritas, digitalizadas através de um aplicativo de celular e enviadas para o meu e-mail. Esses arquivos eram abertos no Acrobat Reader, lidos, comentados e reenviados aos autores para que pudessem apreciá-los. Durante todo período de março até setembro, quando houve o retorno às salas de aula, os problemas relativos à conexão foram constantes, causando desde a falta de sincronia entre a fala e a imagem apresentada até a total interrupção da transmissão. Isso gerou impaciência e desestímulo tanto para professores quanto 
RELATO SOBRE PRÁTICAS PEDAGÓGICAS: PROFESSORES COMPARTILHANDO SUAS EXPERIENNCIAS NO PERÍODO DA PANDEMIA COVID-19
André Machado Barbosa Marco Antonio Serra Viegas Marco Aurelio Carvalho da Costa Regina Lucia Napolitano F. F. Batista

para alunos. Restava claro que não estávamos preparados para uma mudança tão radical exigida em um curtíssimo espaço de tempo.

Por outro lado, a instituição sempre nos orientou a não tornar todo o processo excessivamente extenuante para os alunos e por contiguidade para as famílias, dado que todos passaram a conviver confinados, pelo menos, nos primeiros meses, no mesmo espaço por tempo integral, visto que os pais também tiveram suas tarefas laborais convertidas em atividades remotas.

O isolamento social gerou uma série de contingências domésticas, o que impactou enormemente no propício desenrolar do processo de ensino nos domicílios. Relatos apontam para falta de espaço físico destinado aos estudos, coincidência do horário escolar com o horário laboral dos responsáveis, a necessidade de compartilhar os equipamentos eletrônicos de acesso à internet, enfim, circunstâncias que não tornam o ambiente favorável. Pareceu, contudo, que o problema de maior peso na vida dos estudantes foi a quebra de uma rotina que se organizava em torno da escola enquanto instituição, o que demandava o compromisso com horários e tarefas divididas ao longo do dia. Essa falta ocasionou efeitos deletérios como a alteração dos turnos de descanso, o que elevou o absentismo nas aulas on-line; o aumento da ansiedade, com relatos de acréscimo de peso, acompanhados de sentimentos de melancolia e, em alguns casos, até de depressão.

Ao longo desse período, pelo qual ainda atravessamos, pudemos constatar que não estávamos preparados para uma ruptura tão repentina dos paradigmas que constituíam as bases da nossa forma de ensinar e aprender; contudo, a tecnologia foi capaz de mitigar problemas e indicar soluções, de maneira rápida, para questões que se apresentaram emergenciais. Como saldo, percebeu-se que a escola, como instituição física, ainda é um núcleo fundamental na organização da nossa estrutura social, posto que medeia instâncias que vão além da exclusiva função de transmitir conteúdos previstos em programas pedagógicos. O avanço das tecnologias pode auxiliar enormemente a maneira como propagamos o saber, mas não substituirá a validade das trocas que se estabelecem no convívio físico com o outro. 
RELATO SOBRE PRÁTICAS PEDAGÓGICAS: PROFESSORES COMPARTILHANDO SUAS EXPERIENNCIAS NO PERÍODO DA PANDEMIA COVID-19
André Machado Barbosa Marco Antonio Serra Viegas Marco Aurelio Carvalho da Costa Regina Lucia Napolitano F. F. Batista

\subsection{Professor no Ensino Superior}

Na graduação, foram inúmeras as experiências vivenciadas, boas e ruins, a serem relatadas. Quanto ao aspecto tecnológico, apesar de observar que alguns não tiveram acesso, o triste é ter tido a percepção dos que tiveram o privilégio, mas não souberam aproveitar os potenciais canais disponibilizados. Poderiam, nesse caso, ter potencializado suas habilidades interpessoais, dentre elas, a comunicação, tão importante para o processo de ascensão profissional dos discentes.

Nos dois semestres de 2020, as aulas foram todas ministradas pelas plataformas digitais no modelo híbrido. No primeiro momento, a adaptação requereu dos docentes horas de treinamento no contraturno, o que levou, muitas vezes, à exaustão física e mental. O esgotamento se deu, tendo em vista os treinamentos que eram quase diários, pois tínhamos a necessidade imediata de utilizar as ferramentas nas aulas.

Inicialmente, a preocupação das Instituições de Nível Superior (IES) era com o desenvolvimento de habilidades dos professores em utilizarem apenas o dispositivo para aplicar as aulas online. No segundo momento, logo após a aplicação das primeiras avaliações, começaram a treinar os docentes, com novas metodologias, a fim de tornar as aulas mais dinâmicas por meio de metodologias ativas.

O surpreendente é que, no meio do processo, como nas metodologias ágeis de projetos, foram aplicados modelos de ensino novos, com alteração nos conteúdos educacionais, e práticas pedagógicas. Oportuno, talvez, mas o observável é que foram momentos de muito desgaste físico e emocional que impactaram, de forma negativa, o processo de ensino-aprendizagem, em contraponto, conforme pontua Talbert (2019, p. 91), dado ao fato de haver a necessidade de um planejamento prévio.

O ponto de vista de forma negativa dá-se da compreensão ao fato de os discentes terem sido impactados no período da pandemia. Muitos desistiram devido 
RELATO SOBRE PRÁTICAS PEDAGÓGICAS: PROFESSORES COMPARTILHANDO SUAS EXPERIENNCIAS NO PERÍODO DA PANDEMIA COVID-19
André Machado Barbosa Marco Antonio Serra Viegas Marco Aurelio Carvalho da Costa Regina Lucia Napolitano F. F. Batista

a perdas financeiras e de entes queridos e, também, à dificuldade de assimilar o novo processo de ensino-aprendizagem. Nos que permaneceram, observou-se uma baixa de rendimento, compreensível por tudo que estavam vivenciando de mudanças em todos os aspectos.

Das IES que leciono, uma flexibilizou e manteve os conteúdos educacionais, não houve alteração sequer na ementa, o que trouxe, de certa forma, uma segurança aos discentes. Quanto à prática pedagógica, pouco se alterou, sendo visto como um ponto positivo, já que os estudantes já estavam acostumados àqueles métodos. Apenas o canal de comunicação foi alterado do presencial para o modelo híbrido.

A outra instituição fez mudanças impactantes, das quais consideradas pelos gestores e alguns docentes como profícua. Já os discentes, em sua maioria, apresentaram muitas dificuldades em assimilar conteúdos e o novo método. Cabe salientar que, mesmo diante das dificuldades encontradas, a equipe à qual pertenço nas duas instituições se mostraram proativas e foram bem avaliadas pelos discentes. Isso é um ponto louvável a ser ressaltado, pois aponta como os educadores foram essenciais para esse processo contínuo não ter sido interrompido e provocado maiores impactos na sociedade.

\section{CONSIDERAÇÕES FINAIS}

Ainda estamos atravessando a crise sanitária provocada pela pandemia do COVID-19, sendo necessário todos os dias nos aprimorarmos, tendo em vista os novos rumos que a educação estava e ainda está passando. Nesse sinal de crise ou de mudança (para melhor ou pior), uns agiram negando o problema, outros agiram com pitiatismo e outros agiram de modo tradicionalista.

Aqueles que foram resilientes e tiveram inteligência emocional sobrevivem a quebra de paradigmas. Com isso, a sociedade, de modo geral, pôde constatar que não somos apenas transmissores de conhecimento. Somos subsídios para diminuir as dificuldades existentes na interação discentes com as suas obtenções do saber, 
RELATO SOBRE PRÁTICAS PEDAGÓGICAS: PROFESSORES COMPARTILHANDO SUAS EXPERIÊNCIAS NO PERÍODO DA PANDEMIA COVID-19
André Machado Barbosa Marco Antonio Serra Viegas Marco Aurelio Carvalho da Costa Regina Lucia Napolitano F. F. Batista

auxiliando-os e, muitas vezes, provocando-os a descobrir a utilidade, a função e os objetivos a que se preconiza.

Vivemos na sociedade do conhecimento, sendo assim, tornamo-nos dependentes da informação, para transformá-las em conhecimento aplicado, à qual chamamos de habilidade. Desse modo, os docentes são essenciais em qualquer prática pedagógica, uma vez que são agentes transformadores pelo processo de produção de conhecimento, envolvendo suportes mediadores, tecnológicos ou não, procedimentos que interagem e relações que envolvem docentes, estudantes e conhecimento.

Enfim, devemos estar atentos ao habitual e às atualizações pelas quais as instituições de ensino e a sociedade estão passando. E claro, com o objetivo sempre de oferecer, em nossas práticas pedagógicas, a transmissão do conhecimento de forma eficaz e sermos capazes de desenvolver habilidades e atitudes, o que demonstrará a eficiência de nossas capacidades profissionais.

\section{REFERÊNCIAS}

CARVALHO, Alessandra Aparecida.; BORGES, Raquel Auxiliadora.; AMENO, Viviane Pena Carvalho Silva. Gamificação no processo ensino aprendizagem. In: NEVES, Vander José; MERCANTI, Luiz Bittencourt; LIMA, Maria Tereza. (Org.). Metodologias ativas: perspectivas teóricas e práticas no Ensino Superior. 1ed.Campinas: Pontes Editora, v.1, 2018

CORTELAZZO, Metodologias Ativas e Personalizadas de Aprendizagem. Rio de Janeiro: Alta Books Editora, 2019.

DARLING-HAMMOND, Linda. BRANSFORD, John. Preparando os professores para um mundo em transformação: o que devem aprender e estar aptos a fazer. Porto Alegre: Penso, 2019.

FILATRO, Andrea. Produção de conteúdos educacionais. São Paulo: Saraiva, 2018.

FILATRO, Andrea; CAVALCANTI, Carolina Costa. Metodologias Inov-ativas na educação presencial, a distância e corporativa. 1.ed. São Paulo: Saraiva Educação, 2018. 
RELATO SOBRE PRÁTICAS PEDAGÓGICAS: PROFESSORES COMPARTILHANDO SUAS EXPERIENNCIAS NO PERÍODO DA PANDEMIA COVID-19
André Machado Barbosa Marco Antonio Serra Viegas Marco Aurelio Carvalho da Costa Regina Lucia Napolitano F. F. Batista

LEAL, Edvalda Araújo. MIRANDA, Gilberto José. NOVA, Silvia Pereira de Castro Casa (org.). Revolucionando a sala de aula: como envolver o estudante aplicando as técnicas de metodologias ativas de aprendizagem. 1. ed. [2. reimpr.]. São Paulo: Atlas, 2018.

MARTINO, Luís Mauro Sá. Métodos de pesquisa em Comunicação: projetos, ideias, práticas. Petrópolis, RJ: Vozes, 2018.

MORAN, José. Metodologias ativas para uma aprendizagem mais profunda. 1-25 p. In: Metodologias ativas para uma educação inovadora: uma abordagem teórico-prática. BACICH, Lilian; MORAN, José (org.). Porto Alegre: Penso, 2018.

NEVES, Vander José das; MERCANTI, Luiz Bittencourt; LIMA, Maria Teresa. Metodologias ativas: perspectivas, teorias e práticas do ensino superior. Campinas, SP: Pontes Editores, 2018.

TALBERT, Robert. Guia para utilização da aprendizagem invertida no ensino superior. Porto Alegre: Penso, 2019. 